\title{
Plasma adiponectin levels are correlated with body composition, metabolic profiles, and mitochondrial markers in individuals with chronic spinal cord injury
}

\author{
Laura C. O'Brien ${ }^{1,2} \cdot$ Zachary A. Graham $\mathbb{1}^{3,4} \cdot$ Qun Chen $^{5} \cdot$ Edward J. Lesnefsky ${ }^{2,5,6,7} \cdot$ Christopher Cardozo $^{3,4,8,9}$. \\ Ashraf S. Gorgey (i) $^{1,10}$
}

Received: 29 November 2017 / Revised: 20 February 2018 / Accepted: 23 February 2018 / Published online: 20 March 2018

(c) International Spinal Cord Society 2018

\begin{abstract}
Study design Cross-sectional design.

Objectives This study examined the relationships between circulating adiponectin levels, body composition, metabolic profile, and measures of skeletal muscle mitochondrial enzyme activity and biogenesis.

Settings Clinical Research in a Medical Center.

Methods Plasma adiponectin was quantified in 19 individuals with chronic spinal cord injury (SCI). Body composition was evaluated by dual x-ray absorptiometry and magnetic resonance imaging. Metabolic profile was assessed by basal metabolic rate (BMR), oxygen uptake $\left(\mathrm{VO}_{2}\right)$, and intravenous glucose tolerance testing. Mitochondrial enzyme activity of skeletal muscle was obtained by spectrophotometric assays and peroxisome proliferator-activated receptor gamma coactivator 1-alpha (PGC-1 $\alpha$ ) and 5' AMP-activated protein kinase (AMPK) protein expression was assessed by Western blots.

Results Adiponectin was negatively related to both total and regional fat mass and positively related to lean mass and muscle mass. Furthermore, there were positive relationships between adiponectin and BMR $(r=0.52, P=0.02)$ and $\mathrm{VO}_{2}(r=0.73$, $P=0.01)$. Furthermore, adiponectin was positively related to citrate synthase $(r=0.68, P=0.002)$ and complex III activity $(r=0.57, P=0.02)$. The relationships between adiponectin and body composition remained significant after accounting for age. The relationships between adiponectin, metabolic profile, and markers of mitochondria mass and activity were influenced by age.

Conclusions The study demonstrated that adiponectin is closely related to body composition and metabolic profile in persons with SCI and further supports mechanistic studies suggesting that adiponectin may stimulate mitochondrial biogenesis.
\end{abstract}

Ashraf S. Gorgey

ashraf.gorgey@va.gov

1 Spinal Cord Injury and Disorders, Hunter Holmes McGuire VA Medical Center, Richmond, VA, USA

2 Department of Physiology and Biophysics, Virginia Commonwealth University, Richmond, VA, USA

3 Department of James J. Peters VA Medical Center, Bronx, NY, USA

4 Department of Medicine, Icahn School of Medicine, New York, NY, USA

5 Department of Medicine, Division of Cardiology, Pauley Heart
Center, Virginia Commonwealth University, Richmond, VA, USA

6 Medical Services, Hunter Holmes McGuire VA Medical Center, Richmond, VA, USA

7 Department of Biochemistry and Molecular Biology, Virginia Commonwealth University, Richmond, VA, USA

8 Department of Rehabilitation Medicine, Icahn School of Medicine, New York, NY, USA

9 Department of Pharmacologic Science, Icahn School of Medicine, New York, NY, USA

10 Department of Physical Medicine and Rehabilitation, Virginia Commonwealth University, Richmond, VA, USA 


\section{Introduction}

Adiponectin is a circulating hormone released predominately from adipocytes [1-4]. Paradoxically, levels have shown to be decreased in obese individuals and are negatively related to percent body fat mass [2, 5-7]. Adiponectin levels are also decreased in individuals with type II diabetes and cardiovascular disease $[8,9]$. Previous studies in animal and cell culture models have shown that adiponectin has anti-inflammatory properties and insulinsensitizing effects on muscle and liver [10, 11]. Increasing adiponectin has been shown to reverse insulin resistance and reduce atherosclerosis in animal models $[12,13]$.

One mechanism by which adiponectin exerts beneficial effects may be by increasing mitochondrial bioenergetics $[9,14]$. Mitochondria undergo a cycle of biogenesis, remodeling, and degradation based on the energy needs of the cell. Upstream regulators of mitochondrial biogenesis include 5' AMP-activated protein kinase (AMPK) and peroxisome proliferator-activated receptor gamma coactivator 1 -alpha (PGC-1 $\alpha$ ). Moreover, AMPK is an important sensor of energy balance and phosphorylation of AMPK increases production of PGC-1 $\alpha$ [15]. Treatment of primary human myotubes with adiponectin resulted in increased PGC- $1 \alpha$, fatty acid oxidation, mitochondrial DNA content, and citrate synthase (CS), a marker of mitochondrial mass [9]. Inhibition of AMPK blunted fatty acid oxidation and CS [9, 14]. Similarly, adiponectin signaling has been shown to activate AMPK in vivo and increase skeletal muscle glucose uptake [16]. Lack of skeletal muscle adiponectin signaling resulted in decreased oxidative muscle fibers and exercise intolerance [17]. In humans, mitochondrial DNA copy number was positively related to skeletal muscle adiponectin receptor expression [9].

Persons with spinal cord injury (SCI) are at high risks of developing obesity, type II diabetes and cardiovascular disease [18, 19]. The extensive loss of lean mass and increased fat mass that occurs after injury negatively impacts basal metabolic rate (BMR) and peripheral glucose utilization [20, 21]. Furthermore, the negative changes in body composition and metabolic profile are likely to be impacted by decreased level of physical activity after SCI $[22,23]$. Previous work indicated that serum adiponectin was negatively correlated with body mass index (BMI) and visceral fat in individuals with SCI [24, 25]. One study showed that persons with SCI have a higher level of circulating adiponectin compared to able-bodied controls [24]. The difference in circulating adiponectin was explained by loss in inhibitory effects of sympathetic nervous system. It was also recommended that adipose tissue may secrete different inactive forms of adiponectin that may serve as a protective mechanism of decreased BMR after SCI [24].
Table 1 Physical characteristics of persons with motor complete SCI

Total participants

\section{Demographics}

$\begin{array}{ll}\text { Age (years) } & 36.0 \pm 10.0 \\ \text { BMI }\left(\mathrm{kg} / \mathrm{m}^{2}\right) & 25 \pm 4 \\ \text { TSI (years) } & 8 \pm 8 \\ \text { White, } n(\%) & 14(64 \%)\end{array}$

Injury level

Paraplegia, $n$ (\%), AIS 14 (T4-T11;64\%), AIS A (11) \& AIS B (3)

Tetraplegia, $n(\%)$, AIS 8 (C5-C7; 36\%), AIS A (5) \& AIS B (3)

Values are mean $\pm \mathrm{SD}$

AIS American Spinal Injury Association Impairment Scale, BMI body mass index, TSI time since injury

$n=22$

Recent work indicated that increase in both lean mass and thigh muscle cross-sectional area is associated with increase CS, a marker of mitochondrial density, that was positively related to BMR [26, 27]. The increase in CS was associated with decreased ectopic adiposity and improvement in lipid profile in persons with SCI [26, 27]. However, it is unclear whether circulating adiponectin may trigger increase in CS and subsequent increase in BMR after SCI [25]. The current study investigated the relationships between adiponectin, body composition, metabolic profile, and mitochondrial enzyme activity and biogenesis in individuals with chronic SCI. We hypothesized that circulating adiponectin would be negatively related to measures of body fat and positively related to lean mass and metabolic profile. Furthermore, we hypothesized that mitochondrial enzyme activity and proteins involved in mitochondrial biogenesis would be positively related to adiponectin.

\section{Methods}

\section{Ethical approval}

This study was approved by the McGuire VA Medical Center institutional review board. Participants provided written informed consent as part of a clinical trial, registered at clincialtrials.gov (NCT01652040). Data presented are prior to any intervention.

\section{Participants}

Twenty-two men with motor complete SCI were recruited to participate in the current study. Participants were recruited for a clinical trial investigating the effects of evoked resistance training and testosterone replacement therapy in men with chronic SCI [28]. Persons with motor 
complete SCI were only studied to ensure a homogenous sample and to decrease the inadvertent effects on body composition and metabolic profile. Individuals were between the ages of 18 and 50 and were at least 1-year post injury. The study protocol has been recently published [28] and participants are the same cohort as in previously published studies [26, 27]. Participant characteristics are shown in Table 1.

\section{Metabolic profile assessment}

BMR was measured using a COSMED K4b2 (Cosmed USA Inc., Chicago, IL) with canopy after overnight fast for 10-12 $\mathrm{h}$ followed by an intravenous glucose tolerance test as previously described [27, 29, 30]. After calibration, the participants were gently awakened in the morning and a see-through canopy was placed over the participant's head while lying flat with a clear instruction to lay still during the 20-min test. The first $5 \mathrm{~min}$ of the test was disregarded and the last $15 \mathrm{~min}$ were analyzed after ensuring a steady state has been reached for oxygen uptake $\left(\mathrm{VO}_{2}\right), \mathrm{VCO}_{2}$, and respiratory exchange ratio. The Weir equation was used to calculate BMR [29]. Blood was collected and sent out to a clinical diagnostic lab to determine the fasting lipid profile, plasma glucose, and glycated hemoglobin (HbA1c). Insulin sensitivity $\left(S_{\mathrm{i}}\right)$ and glucose effectiveness $\left(S_{\mathrm{g}}\right)$ were determined using the MinMod software (MinMod Inc., Pasadena, CA) [31]. Three $S_{\mathrm{i}}$ values were excluded because they were outside the physiological range. Fasting plasma glucose and $\mathrm{HbA} 1 \mathrm{c}$ values from one individual were unavailable due to lab error.

$\mathrm{VO}_{2}$ was measured in a subset of participants $(n=15)$ during functional electrical stimulation cycling on a RTI300 bike (Restorative Therapies) using a COSMED K4b2 [32]. Electrical stimulation was applied bilaterally to the quadriceps, hamstrings, and gluteal muscles to achieve exercise in a cyclical pattern [28, 32]. Stimulation parameters were set at $30 \mathrm{~Hz}$, pulse duration at $450 \mu \mathrm{s}$, and current amplitude ranged from 100 to $140 \mathrm{~mA}$ [32]. After a 3-min resting period, participant cycled for a 3-min warmup period with the motor on. The testing period involved cycling at speed of 40-45 revolutions per minute (RPM) and manually increasing the resistance by $2 \mathrm{Nm}$ every $2 \mathrm{~min}$ with the motor off until fatigue (i.e., speed below 18 RPM). This was followed by a cool down period of $1 \mathrm{~min}$ and a 5min period of rest as previously described [32]. Blood pressure and heart rate were monitored every 2-3 min to protect against episodes of autonomic dysreflexia that may occur especially in those with high level of injury. Data are then normalized to body mass $(\mathrm{ml} / \mathrm{min} / \mathrm{kg})$.

\section{Body composition}

Total body and regional dual-energy x-ray absorptiometry (DXA) scans were performed with a Lunar Prodigy Advance scanner (Lunar Inc., Madison, WI) by a trained operator to measure lean mass and fat mass. Once participant was transferred to the DXA table, both legs were strapped proximal to the knee joints and around both feet to avoid any movement artifact due to muscle spasms during scanning. Participants were allowed $20 \mathrm{~min}$ in a supine position before conducting the scan to minimize the effects of fluid shift on body composition assessment. The RMS$\mathrm{CV} \%$ for legs and total body for same day repeated scans was $2.7 \%$ and $2.3 \%$, respectively [33]. Weight and height were determined as previously described [26-30].

Transaxial magnetic resonance imaging was captured to measure whole thigh skeletal muscle and knee extensor cross-sectional area (slice thickness was $8 \mathrm{~mm}$ and interslice space was $16 \mathrm{~mm}$ apart) from the hip joint to the knee joint as previously described [23, 34]. Analysis was performed using Win-vessel software (Ronald Meyer, MSU) by an experimenter blinded to the experimental conditions [23, 34]. Data presented are the average of 12-15 slices from the right leg. MRI data were not available from one participant with a previous gunshot wound. The magnetic field may possibly cause movement of the sharpeners in the spinal canal and may lead to further damage.

\section{Adiponectin}

Blood samples were obtained between 6:00 and 7:00 AM after an overnight fast and plasma samples were stored at $-70{ }^{\circ} \mathrm{C}$ until batch analysis. Total adiponectin was quantified in duplicate by colorimetric enzyme-linked immunosorbent assay (ELISA; Alpco Diagnostics, Salem, NH, USA). This assay has a detection limit of $0.034 \mathrm{ng} / \mathrm{ml}$. Blood samples from three individuals were unavailable for analysis, because of shortage in the blood volumes drawn from these participants Adiponectin was expressed in absolute form $(\mathrm{ng} / \mathrm{ml})$ and relative to body weight, lean mass, and fat mass $(\mathrm{ng} / \mathrm{ml} / \mathrm{kg}$ ) to control for variability relative to body size and body composition compartments.

\section{Enzyme activities}

Muscle biopsies were collected from the vastus lateralis of the right leg using a 14-gauge tru-cut ${ }^{\mathrm{TM}}$ needle under local anesthesia ( $2 \%$ lidocaine). Samples were immediately frozen in liquid nitrogen and stored at $-70{ }^{\circ} \mathrm{C}$ until analysis. A portion of the sample $(\sim 10-25 \mathrm{mg})$ was removed of visible connective and adipose tissue and homogenized in ice cold buffer containing $220 \mathrm{mM}$ mannitol, $70 \mathrm{mM}$ sucrose, $5 \mathrm{mM}$ MOPS, $2 \mathrm{mM}$ EDTA, with cOmplete ${ }^{\mathrm{TM}}$ protease inhibitor 
cocktail (Sigma-Aldrich), $\mathrm{pH}$ 7.4. The homogenate was centrifuged at $371 \mathrm{~g}\left(5 \mathrm{~min}, 4^{\circ} \mathrm{C}\right)$ and the supernatant was used for analysis. Protein concentration was determined and samples were solubilized in $1 \%$ potassium cholate. Assays were completed on the same day as homogenization. CS and complex III (CIII) activity were measured spectrophotometrically in duplicate or triplicate as previously described [26, 27, 35]. Insufficient muscle tissues precluded analysis of four samples for CS analysis and seven samples for CIII analysis.

\section{Western blot}

Muscle tissue was homogenized in RIPA buffer with containing protease and phosphatase inhibitors (Halt Protease and Phosphatase, ThermoScientific) using an electric tissue homogenizer [30]. The homogenate was briefly sonicated and centrifuged for $10 \mathrm{~min}$ at $4{ }^{\circ} \mathrm{C}$ at $10,000 \mathrm{~g}$. A microBCA kit (Thermo Scientific) was used to measure the protein content of the supernatant using bovine serum albumin as the standard. Samples were mixed 1:1 in $2 \times$ Laemlli sample buffer with 2-mercaptoethanol and boiled for $3 \mathrm{~min} ; 50 \mu \mathrm{g}$ of protein was loaded onto a $10 \%$ polyacrylamide gel and run for $60 \mathrm{~min}$ at $185 \mathrm{~V}$. Protein was electrophoretically transferred to a PVDF membrane and stained with Ponceau $\mathrm{S}$ to visualize protein loading. The membrane was destained with a $10-15$ min wash in Tris-buffered saline with $0.05 \%$ Tween 20 (TBS-T) and blocked in a solution of 5\% milk and TBS-T for $1 \mathrm{~h}$ followed by overnight incubation at $4{ }^{\circ} \mathrm{C}$ with primary antibody diluted 1:1000 in $1 \%$ milk and TBST. Primary antibodies included PGC1 $\alpha$ (sc-13067; Santa Cruz Biotechnology), total AMPK (\#2532; Cell Signaling), and AMPKT172 (\#50081; Cell Signaling). Membranes were washed in TBS-T with three 10-min washes, then incubated for an hour with a horseradish peroxidaseconjugated secondary antibody (1:2000, Cell Signaling). The membranes were washed as mentioned above, then incubated for $5 \mathrm{~min}$ with a horseradish peroxidase chemiluminescence detection reagent (ECL Prime: Amersham) and digitaly imaged (A600; Amersham) [30]. Densitometry was analyzed with iQuant software (Amersham). AMPK data are shown as the ratio of phosphorylated AMPK/total AMPK.

\section{Statistical analyses}

Data are expressed as mean \pm standard deviation. Data are checked for normal distribution using Shapiro-Wilk test and then log-transformed if needed $(P<0.05)$ prior to any statistical analyses. Independent two-tailed $t$-tests were used to evaluate differences in adiponectin based on SCI characteristics including level of injury, age ( $\geq 40$ or $<40$ years), time since injury ( $\geq 6$ or $<6$ years), BMI $(>25$ or $<25$ ), and
Table 2 Body composition and metabolic outcomes of persons with motor complete SCI

\begin{tabular}{|c|c|}
\hline Outcome variables & Mean \pm SD \\
\hline \multicolumn{2}{|l|}{ Body composition } \\
\hline Total \%Fat & $32.6 \pm 9.7, n=22$ \\
\hline Total LM (kg) & $49.5 \pm 7.5, n=22$ \\
\hline Leg $\%$ Fat & $34.1 \pm 9.6, n=22$ \\
\hline Leg LM (kg) & $14.2 \pm 3.6, n=22$ \\
\hline$\%$ IMF (thigh) & $14.1 \pm 8.8, n=20$ \\
\hline Thigh muscle CSA $\left(\mathrm{cm}^{2}\right)$ & $82.2 \pm 21.2, n=20$ \\
\hline Knee extensor CSA $\left(\mathrm{cm}^{2}\right)$ & $37.1 \pm 10.4, n=20$ \\
\hline \multicolumn{2}{|l|}{ Metabolic profile } \\
\hline Fasting glucose (mg/dl) & $107.1 \pm 13.0, n=21$ \\
\hline HbA1c $(\%)$ & $5.35 \pm 0.45, n=21$ \\
\hline$\left.S_{\mathrm{i}}(/(\mathrm{mU} / \mathrm{L}) / \mathrm{min})\right)$ & $8.6 \pm 6.3, n=19$ \\
\hline$S_{\mathrm{g}}\left(10^{-2} / \mathrm{min}\right)$ & $2.01 \pm 0.88, n=22$ \\
\hline BMR (kcal/day) & $1547 \pm 177, n=22$ \\
\hline $\mathrm{VO}_{2}(\mathrm{ml} / \mathrm{min} / \mathrm{kg})$ & $7.04 \pm 3.18, n=15$ \\
\hline \multicolumn{2}{|l|}{ Mitochondrial enzyme activity } \\
\hline $\mathrm{CS}$ activity (nmol/min/mg) & $52.7 \pm 28.5, n=18$ \\
\hline CIII activity (nmol/min/mg) & $34.2 \pm 18.0, n=15$ \\
\hline Adiponectin (ng/ml) & $3892.6 \pm 1493.9 ; n=19$ \\
\hline Adiponectin/body weight (ng/ml/kg) & $52.2 \pm 23.3 ; n=19$ \\
\hline Adiponectin/ lean mass (ng/ml/kg) & $78.6 \pm 26 ; n=19$ \\
\hline Adiponectin/ fat mass (ng/ml/kg) & $207 \pm 175 ; n=19$ \\
\hline $\begin{array}{l}\text { PGC-1 } \alpha \text { Protein expression (arbitrary } \\
\text { units) }\end{array}$ & $\begin{array}{l}(744.2 \pm 1269) \times 10^{-4} ; n= \\
18\end{array}$ \\
\hline Phospho-AMPK & $\begin{array}{l}(360.2 \pm 284) \times 10^{-4} \\
\mathrm{n}=18\end{array}$ \\
\hline Phospho AMPK/ total AMPK & $1.23 \pm 1.30 ; \mathrm{n}=18$ \\
\hline
\end{tabular}

Values are means \pm SD

$n$ number of subjects, $L M$ lean mass, IMF intramuscular fat, CSA cross-sectional area, $K E$ knee extensor, $H b A l c$ glycated hemoglobin, $S_{\mathrm{i}}$ insulin sensitivity, $S_{\mathrm{g}}$ glucose effectiveness, $B M R$ basal metabolic rate, $\mathrm{VO}_{2}$ oxygen uptake, $\mathrm{CS}$ citrate synthase, $\mathrm{CIII}$ complex III

ethnicity (African-American vs. Caucasian). Bivariate Pearson correlations were used to assess the relationship between lipid and metabolic variables and plasma adiponectin. Partial correlations were conducted to consider age as a confounding variable. Statistical significance was set at $P<0.05$. All statistical analyses were performed using SPSS version 23 (Armonk, NY).

\section{Results}

\section{Participant characteristics}

Participant demographics are shown in Table 1. Participants ranged in age from 18 to 50 with a mean age of $36 \pm 10$ years and were an average of $8 \pm 8$ years post injury (range 
from 1 to 28). All participants were motor complete (no motor function below the level of injury). Table 2 shows the values for body composition, metabolic profile, skeletal muscle mitochondrial enzyme activities, and absolute adiponectin as well as adiponectin adjusted to body weight, lean mass, and fat mass. Values of PGC-1 $\alpha$, phosphorylated AMPK, and phosphorylated AMPK to total AMPK ratio are also listed.

\section{Adiponectin and $\mathrm{SCl}$ characteristics}

There was a trend $(P=0.06)$ of greater adiponectin between individuals age $<40$ years $(n=13 ; 4318 \pm 1474.5 \mathrm{ng} / \mathrm{dl})$ and age $\geq 40$ years $(n=6 ; 2972 \pm 1153 \mathrm{ng} / \mathrm{dl})$. Caucasians $(n=$ $12 ; 4478 \pm 1407 \mathrm{ng} / \mathrm{dl})$ have greater $(P=0.007)$ adiponectin compared to African-Americans $(n=7 ; 2889 \pm 1097 \mathrm{ng} / \mathrm{dl})$ men with SCI. There were no effects of level of injury (tetraplegia vs. paraplegia), time since injury $(\geq 6$ or $<6$ years), AIS classification (A vs. B), and BMI ( $\geq 25$ or $<25$ $\mathrm{kg} / \mathrm{m}^{2}$ ) on adiponectin level. Adiponectin was negatively related to age $(r=-0.62, P=0.005)$ and adiponectin adjusted to fat mass was negatively related to age ( $r=-0.62, \quad P=0.004)$ and body weight $(r=-0.48$, $P=0.035)$. Time since injury was not related to adiponectin $(r=-0.2, P=0.2)$.

\section{Relationship between adiponectin and body composition}

Total \%fat $(r=-0.71, P=0.001)$, leg $\%$ fat $(r=-0.65, P$ $=0.003)$, and trunk \%fat $(r=-0.75, P<0.0001)$ were negatively related to adiponectin levels (Fig. 1a). Adjusted adiponectin demonstrated similar relationships with total $\%$ fat $(r=-0.63$ to $-0.89 ; P \leq 0.001-0.004), \operatorname{leg} \%$ fat $(r=$ -0.53 to $-0.83 ; P<0.0001-0.018)$, and trunk \%fat $(r=$ -0.70 to $-0.92 ; P<0.0001-0.001)$. There was a positive relationship between adiponectin and total lean mass $(r=$ $0.62, P=0.005)$ and as well as leg lean mass $(r=0.61, P$ $=0.006$; Fig. 1b). Similarly, there was a positive relationship between adiponectin and thigh muscle CSA $(r=0.58$, $P=0.011)$ as well as knee extensor CSA $(r=0.64, P=$ 0.005 ; Fig. 2a). There was a negative relationship between percent intramuscular fat (\%IMF) and adiponectin $(r=$ $-0.66, P=0.003$; Fig. 2b).

The relationships between adiponectin and total \%fat, total lean mass, leg \%fat, and leg lean mass remained significant when partial correlations were run to account for age as a confounding variable (Table 3). Similarly, there was a trend for a relationship between adiponectin and thigh muscle CSA ( $r=0.43, P=0.09)$ and adiponectin and knee extensor CSA ( $r=0.45, P=0.07)$ when age was accounted for. However, the relationships between adiponectin and $\%$ IMF were no longer significant after accounting for age.

\section{Relationship between adiponectin and metabolic profile}

As shown in Fig. 3, BMR $(r=0.52, P=0.02$; Fig. 3a) and $\mathrm{VO}_{2}(r=0.73, P=0.01$; Fig. $3 \mathrm{~b}$ ) were positively related to adiponectin. No significant relationships were observed between adiponectin and fasting glucose, HbA1c, $\mathrm{Si}, \mathrm{Sg}$, or lipid profile. A trend toward significance remained between BMR and adiponectin when age was taken into consideration ( $r=0.46, P=0.06$; Table 3). However, the relationship with $\mathrm{VO}_{2}$ was no longer significant after accounting for age.

\section{Relationship between adiponectin and markers of mitochondrial mass and biogenesis}

Adiponectin was positively related to CS $(r=0.68$, $P=0.002)$ and CIII activity $(r=0.57, P=0.02)$ (Fig. 4). Similarly, there was a trend for a positive relationship between adiponectin and protein levels of PGC- $1 \alpha$ ( $r=0.46, P=0.06$ ); this relationship is no longer valid after accounting for the non-normal distribution pattern of PGC-1 $\alpha$. There was also no significant relationship between adiponectin and CIII normalized to CS. After accounting for age, trend toward significance remained between CS and adiponectin $(r=0.48, P=0.05)$ but not PGC-1 $\alpha$ ). There was also a trend of adiponectin adjusted to lean mass to PGC-1 $\alpha(r=0.47, P=0.07)$ and negative relationship between adiponectin and phosphorylated AMPK $(r=-0.59, P=0.019)$.

\section{Discussion}

The relationships between serum adiponectin levels, glucose levels and parameters of muscle size, exercise capacity, BMR, and muscle mitochondrial density and activity were examined in persons with SCI. Serum adiponectin levels were inversely related to fat with good concordance for this relationship between whole body fat mass, regional fat mass, and IMF. Similarly, adiponectin levels were positively related to total and leg lean mass, thigh muscle CSA, and knee extensor CSA, again demonstrating good concordance between multiple measures of skeletal muscle and adiponectin. A relationship between adiponectin and BMR, which is greatly impacted by muscle mass and reduced after SCI. An intriguing additional finding was the relationships between adiponectin, $\mathrm{VO}_{2}$, and levels of $\mathrm{CS}$ and CIII which may reflect an effect of adiponectin on mitochondrial biogenesis or health. Relationships between adiponectin and body composition remained significant after accounting for age. However, relationships between adiponectin and metabolism as well as mitochondrial parameters were influenced by age. 

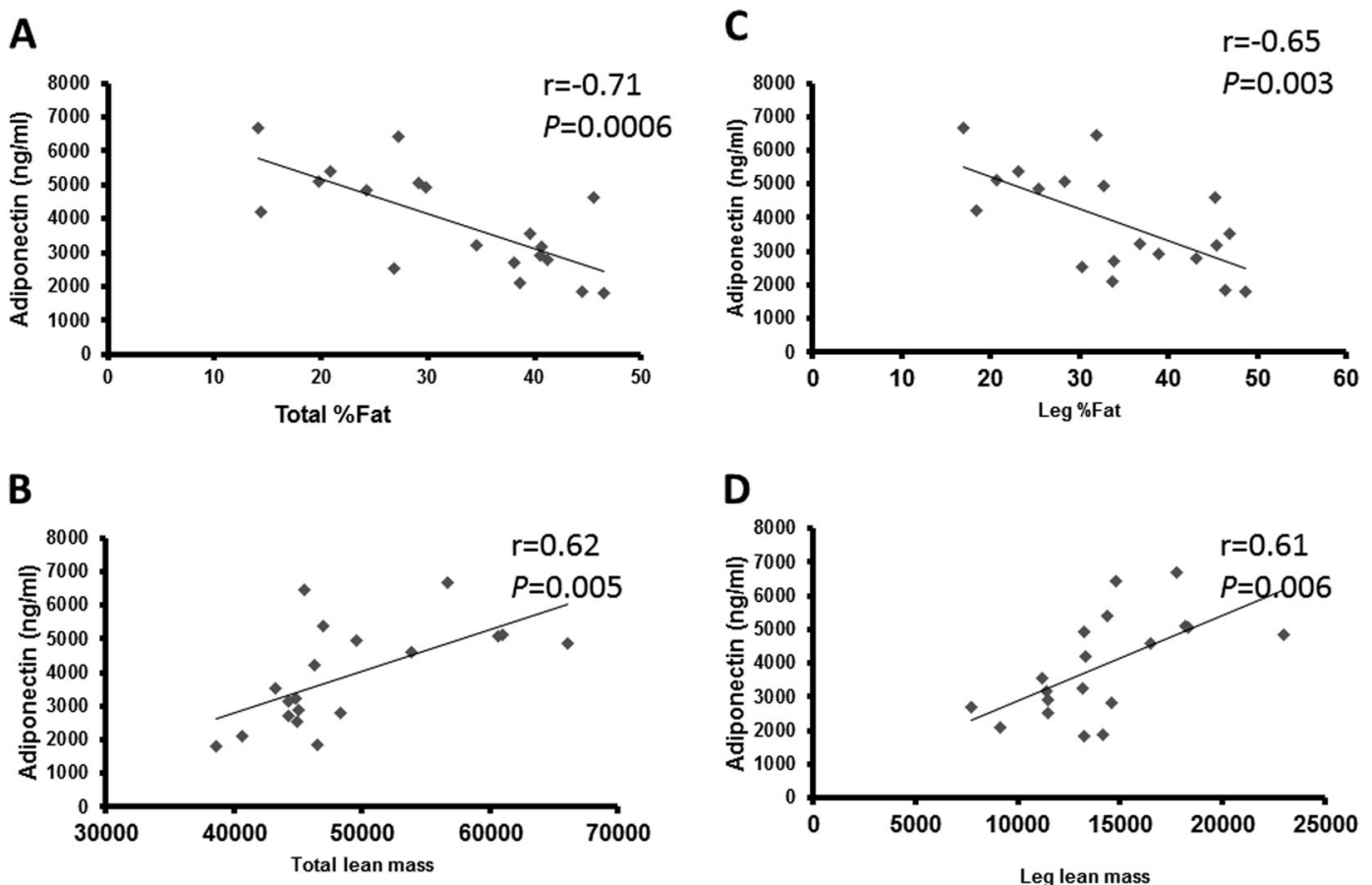

Fig. 1 Total and leg \%fat (a and c, respectively) and LM (b and d, respectively) related to adiponectin in 19 men with SCI

\section{Adiponectin and body composition}

Persons with SCI at heightened risk for obesity, insulin resistance and diabetes. A common and vexing secondary complication of SCI is the constellation of changes affecting metabolism that include a dramatic increase in body fat mass, visceral adiposity, fatty liver, and elevation of a variety of cytokines [36-38]. Given that muscle is responsible for at least $60 \%$ of insulin-mediated glucose uptake, skeletal muscle atrophy after SCI accompanied with decreased mitochondrial density and activity may alter intracellular signaling [26, 27, 30]. Our understanding of the cellular and molecular mechanisms responsible for these alterations is incomplete. No prior work examined the relationships between adiponectin levels and muscle mass or mitochondria after SCI. Thus, our findings support a new role for adiponectin in regulating properties of skeletal muscle below the level of injury. The relationship between adiponectin and body composition are consistent with previous reports in able bodied individuals 6 and those with SCI $[23,24]$. Compared to previous work that used BMI [23], we have performed body composition assessment using both DXA and MRI to accurately determine the relationships between adiponectin and total as well as sublesional fat mass or lean mass. BMI underestimates the percentage of fat mass in persons with SCI. Adiponectin was related to regional measurements of leg fat and lean mass measured by both DXA and MRI. We sought to investigate the relationships with body composition, because of the extreme level of muscle atrophy accompanied with infiltration of IMF below the level of injury. The inverse relationship between adiponectin and IMF is consistent with previous studies showing that adiponectin decreased triglyceride content in muscle [12, 39]. Decreased IMF may be due to increased muscle fatty acid oxidation induced by adiponectin [15]. This is in line with our recent findings that mitchondrial mass and activity was negatively related to $\operatorname{IMF}[26,27]$.

\section{Adiponectin and metabolic profile}

Consistent with the observations in body composition, a positive relationship was seen between adiponectin, BMR, and $\mathrm{VO}_{2}$. Contrasting to previous studies that used armcycling ergometer [30, 40], we have used FES-cycling to determine $\mathrm{VO}_{2}[32,41]$. The limitation of this approach is that attaining peak $\mathrm{VO}_{2}$ may be limited by peripheral neuromuscular fatigue occurred during electrical stimulation [41]. However, this approach is beneficial in grouping both individuals with tetraplegia and paraplegia without considering the discrepancy in upper extremity strength. The findings are in contrast to a previous study that found a negative relationship between circulating adiponectin and metabolic rate $[39,42]$. However, the difference between the current study and that of Ruige et al. [42] with regard to the methods for measuring body composition (bio-electrical impedance vs. DXA) and adiponectin, as well as participant demographics (able-bodied vs. SCI), makes it difficult to compare the two 

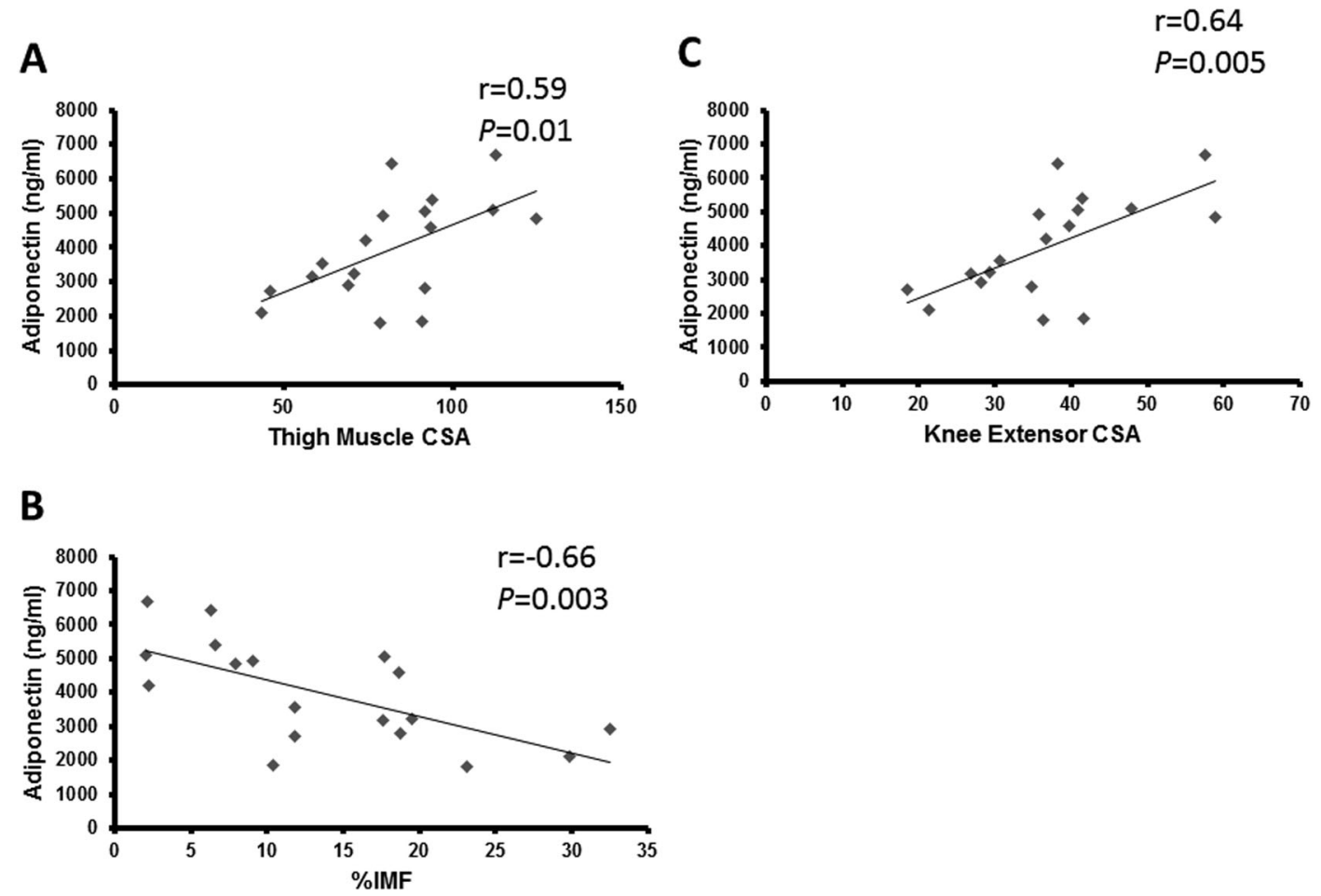

Fig. 2 Adiponectin related to a whole thigh muscle cross-sectional area (CSA), b knee extensor (KE) CSA ( $n=18)$, and $\mathbf{c} \%$ intramuscular fat (\% IMF)

Table 3 Correlation coefficients for adiponectin, body composition, and metabolic profile in persons with motor complete SCI

\begin{tabular}{lll}
\hline & Pearson $(r)$ & Partial (age-adjusted) \\
\hline Total \%fat & $-0.71^{* *}$ & $-0.56^{*}$ \\
Total lean mass & $0.62^{* *}$ & $0.59^{*}$ \\
Trunk \%fat & $-0.75^{* *}$ & $-0.57^{*}$ \\
Leg \%fat & $-0.65^{* *}$ & $-0.59^{*}$ \\
Leg lean mass & $0.61^{* *}$ & $0.52^{*}$ \\
\%IMF & $-0.66^{* *}$ & -0.34 \\
Thigh muscle CSA & $0.59^{*}$ & $0.43^{\wedge}$ \\
Knee extensor CSA & $0.64^{* *}$ & $0.45^{\wedge}$ \\
$\mathrm{BMR}$ & $0.52^{*}$ & $0.46^{\wedge}$ \\
VO & $0.63^{*}$ & 0.29 \\
$\mathrm{CS}$ & $0.68^{* *}$ & $0.48^{\wedge}$ \\
$\mathrm{CIII}$ & $0.57^{*}$ & 0.38 \\
\hline
\end{tabular}

$\overline{I M F}$ intramuscular fat, $C S A$ cross-sectional area, $B M R$ basal metabolic rate, $V O_{2}$ oxygen uptake, $C S$ citrate synthase, $C I I I$ complex III

$* P<0.02 ; * * P<0.0001 ;{ }^{\wedge} P>0.05-0.07$

studies. Moreover, Rugie et al. [42] adjusted BMR to fat-free mass before determining the relationship with adiponectin compared to the current work. Similar to previous studies, steady state was reached within $5 \mathrm{~min}$ from starting the test and the respiratory exchange ratio was within $0.7-0.9$ with coefficient of variations of less than $10 \%$ for both $\mathrm{VO}_{2}$ and $\mathrm{VCO}_{2}$ across the last $15 \mathrm{~min}[29,43]$. Another study showed a positive relationship between mean $\mathrm{VO}_{2}$ and adiponectin in able-bodied individuals [44]. The significance of these findings may rely on the fact that persons with SCI experience diminished BMR that is likely to expose them to increased obesity [36, 37].

\section{Adiponectin and protein expression}

Important determinants of mitochondrial biology include levels and activation of PGC- $1 \alpha$ and activity of AMPK. Levels of PGC-1 $\alpha$ were very low in the majority of subjects, consistent with prior data in rodents following complete spinal cord transection [45]. In subjects for whom higher PGC-1 $\alpha$ levels were observed these appeared to correlate positively with adiponectin raising intriguing questions about the underlying mechanisms. Skeletal muscle expresses adiponectin receptors and adiponectin is well known to increase insulin action and to activate AMPK [12, 16]. AMPK is turn is a potent regulator of cellular metabolism which activates PGC- $1 \alpha$, regulates fatty acid metabolism and insulin action, and drives mitochondrial biogenesis in tissues including skeletal muscle [15]. The relationship between adiponectin and phosphorylated AMPK may suggest that adiponectin regulates the interplay between AMPK and PGC-1 $\alpha$. An increase in mitochondrial function may underlie the positive relationship between adiponectin and BMR. Activation of AMPK by adiponectin is a transient 

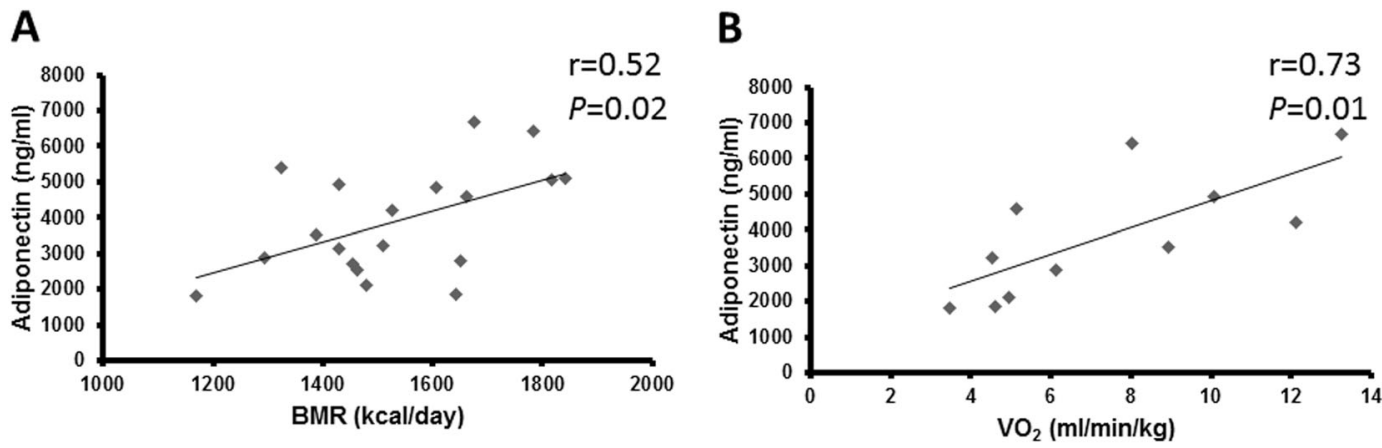

Fig. 3 Basal metabolic rate (a BMR; $n=19)$ and oxygen uptake $\left(\mathbf{b} \mathrm{VO}_{2} ; n=11\right)$ related to adiponectin in individuals with SCI
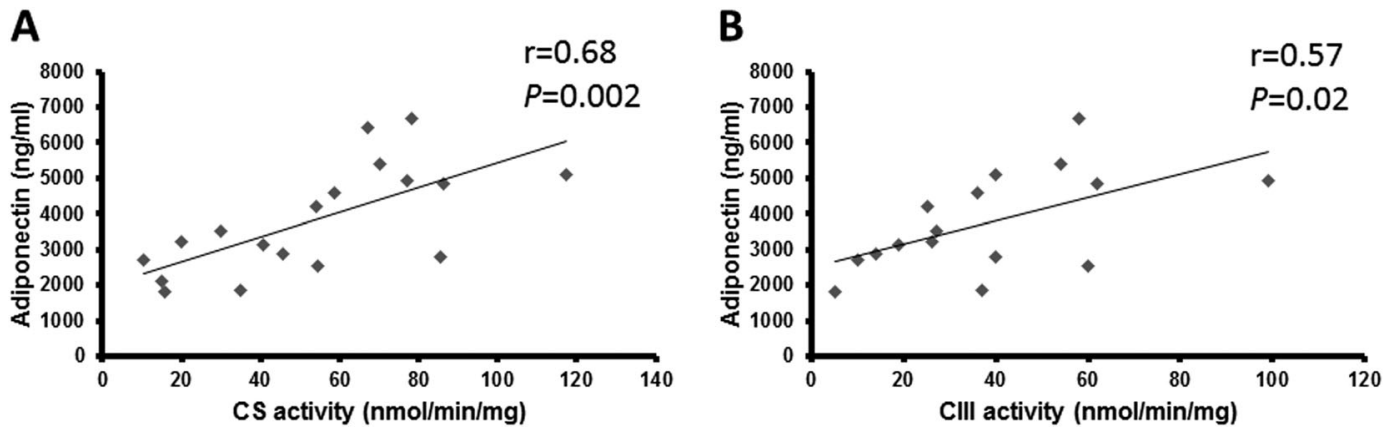

Fig. 4 Skeletal muscle citrate synthase (a CS; $n=18$ ) and complex III (b CIII; $n=16$ ) activity related to adiponectin in individuals with SCI

event, making it difficult to study in vivo. Peak AMPK activation was observed $20-60 \mathrm{~min}$ after adiponectin treatment of C2C12 myoblasts [46] and isolated human skeletal muscle preparations [47] and $5 \mathrm{~min}$ in vivo [16]. A trend for non-significant relationship was observed between adiponectin and another upstream regulator of mitochondrial biogenesis, PGC-1 $\alpha$, in the current study. However, there was considerable variability in PGC- $1 \alpha$ protein expression between individuals. The current study only measured total PGC- $1 \alpha$; however, there are a number of PGC- $1 \alpha$ isoforms that have different functions in skeletal muscle [48]. Specifically, PGC- $1 \alpha 4$ is associated with skeletal muscle hypertrophy but not mitochondrial biogenesis [49]. Furthermore, PGC- $1 \alpha$ activity is modulated by acetylation, ubiquitylation, and phosphorylation and the current study did not investigate these post translational modifications. Even so, the current results suggest a moderate relationship between PGC-1 $\alpha$ and adiponectin which may inform future studies. Further research will be required to unravel how adiponectin levels may contribute to insulin resistance or diabetes in persons with SCI.

\section{Significance of the work and future implications}

The current findings have significant clinical relevance to persons with SCI. Prevalence of obesity, impaired glucose tolerance, insulin resistance and are at a heightened and alarming rate after SCI [37]. Along with decreased level of physical activity, persons with SCI experienced reduced BMR that is likely to be a predisposing factor for several comorbidities. Previous work demonstrated that the frequency and the intensity of the exercise paradigms may not be sufficient to counterbalance several of the health-related comorbidities after SCI. Exercise induced muscle hypertrophy may be a potent stimulus that is associated with increase of circulating plasma adiponectin. The circulating adiponectin will be associated with downstream stimulation of PGC-1 alpha that will lead to increase in body mitochondrial biogenesis and subsequent increase in BMR. The findings may recommend potential pathways that are likely to be used as a countermeasure to resolve this expensive and time-consuming problems. Therefore, recommending pathways that are likely to be target in future pharmaceutical or rehabilitation intervention are of paramount significance to this clinical population.

\section{Limitations}

A major limitation of the current study is that only total adiponectin was measured. Adiponectin exists in multiple forms, only some of which are biologically active [50]. Similarly, only total PGC- $1 \alpha$ was measured. Furthermore, 
the small sample size and inclusion of only men may limit the generalization of the current findings. Future studies may investigate various forms of adiponectin as well as PGC- $1 \alpha$ isoforms in a larger sample size of both men and women with SCI. The current findings indeed do not support causality but it can be simply used to drive future mechanistic research hypothesis that investigate the role of adiponectin on mitochondrial health and BMR after SCI. In the current study, we did not have a matched control group. Previous studies comparing adiponectin levels in individuals with spinal cord compared to able bodied individuals suggests a trend for higher adiponectin levels after SCI [24]; however, these results were not statistically significant. The primary research hypothesis was aimed to investigate the relationships between adiponectin and possible mechanistic associations with BMR.

\section{Conclusions}

This study provides preliminary evidence for a link between adiponectin and metabolic health as demonstrated by increase in BMR and mitochondrial enzyme activity in individuals with SCI. The preliminary evidence presented above supports a negative association between adiponectin and body fat mass, and a positive association with muscle size and mitochondrial parameters after. That is accompanied with concomitant increase in $\mathrm{BMR}$ and $\mathrm{VO}_{2}$. The links between adiponectin levels and the size of paralyzed muscle suggest previously unknown effects of this hormone on muscle homeostasis. These results are consistent with the hypothesis that adiponectin stimulates mitochondrial biogenesis through PGC- $1 \alpha$. Given the critical role of muscle in insulin action, the marked atrophy of muscle below the level of injury, the numerous positive effects adiponectin on metabolism, there is a strong impetus to more fully understand the role of adiponectin on paralyzed muscles after SCI.

Acknowledgements We would like to thank the Hunter Holmes McGuire Medical Center for allowing us the opportunity to conduct clinical research and to all of our participants. We would like also to thank Refka Khalil for research coordination and to Jeremy Thompson and Ying $\mathrm{Hu}$ for technical assistance.

Funding Grants: This work was supported by the Office of Research and Development, Medical Research Service, Department of Veterans Affairs grant \# B7867-W and grant \# B-2020-C.

\section{Compliance with ethical standards}

Conflict of interest The authors declare that they have no conflict of interest.

\section{References}

1. Scherer PE, Williams S, Fogliano M, Baldini G, Lodish HF. A novel serum protein similar to c1q, produced exclusively in adipocytes. J Biol Chem. 1995;270:26746-9.

2. Hu E, Liang P, Spiegelman BM. Adipo Q is a novel adiposespecific gene dysregulated in obesity. J Biol Chem. 1996;271:10697-703.

3. Maeda K, Okubo. K, Shimomura I, Funahashi T, Matsuzawa Y, Matsubara K. cDNA cloning and expression of a novel adipose specific collagen-like factor, apm1 (adiposemost abundant gene transcript Biochem. Biophys Res Commun. 1996;221:286-9.

4. Nakano Y, Tobe T, Choi-Miura NH, Mazda T, Tomita M. Isolation and characterization ofgbp28, a novel gelatin-binding protein purified from human plasma. J Biochem. 1996;120:803-12.

5. Arita Y, Kihara S, Ouchi N, Takahashi M, Maeda K, Miyagawa J, et al. Paradoxical decrease of an adipose-specific protein, adiponectin, in obesity. Biochem Biophys Res Commun. $1999 ; 425: 560-4$.

6. Weyer C, Funahashi T, Tanaka S, Hotta K, Matsuzawa Y, Pratley RE, et al. Hypoadiponectinemia in obesity and type 2 diabetes: close association with insulin resistance and hyperinsulinemia. $\mathrm{J}$ Clin Endocrinol Metab. 2001;86:1930-5.

7. Rasmussen MS, Lihn A, Pedersen SB, Bruun J, Rasmussen M, Richelsen B. Adiponectin receptors in human adipose tissue: effects of obesity, weight loss, and fat depots. Obes Res. 2006;14:28-35.

8. Hotta K, Funahashi T, Arita Y, Takahashi M, Matsuda M, Okamoto $\mathrm{Y}$, et al. Plasma concentrations of a novel, adipose-specific protein, adiponectin, in type 2 diabetic patients. Arterioscler Thromb Vasc Biol. 2000;20:1595-9.

9. Civitarese AE, Jenkinson CP, Richardson D, Bajaj M, Cusi K, Kashyap S, et al. Adiponectin receptors gene expression and insulin sensitivity in non-diabetic Mexican Americans with or without a family history of Type 2 diabetes. Diabetologia. 2004;47:816-20.

10. Yamauchi T, Nio Y, Maki T, Kobayashi M, Takazawa T, Iwabu $\mathrm{M}$, et al. Targeted disruption of adipor1 and adipor2 causes abrogation of adiponectin binding and metabolic actions. Nat Med. 2007;13:332-9.

11. Nawrocki AR, Rajala MW, Tomas E, Pajvani UB, Saha AK, Trumbauer ME, et al. Mice lacking adiponectin show decreased hepatic insulin sensitivity and reduced responsiveness to peroxisome proliferator-activated receptor gamma agonists. Biol Chem. 2006;281:2654-60.

12. Yamauchi T, Kamon J, Waki H, Terauchi Y, Kubota N, Hara K, et al. The fat-derived hormone adiponectin reverses insulin resistance associated with both lipoatrophy and obesity. Nat Med. 2001;7:941-6.

13. Okamoto Y, Kihara S, Ouchi N, Nishida M, Arita Y, Kumada M, et al. Adiponectin reduces atherosclerosis in apolipoprotein e-deficient mice. Circulation. 2002;106:2767-70.

14. Yoon MJ, Lee GY, Chung JJ, Ahn YH, Hong SH, Kim JB. Adiponectin increases fatty acid oxidation in skeletal muscle cells by sequential activation of AMP-activated protein kinase, p38 mitogen-activated protein kinase, and peroxisome proliferatoractivated receptor alpha. Diabetes. 2006;55:2562-70.

15. Jäger S, Handschin C, St-Pierre J, Spiegelman BM. AMPactivated protein kinase (AMPK) action in skeletal muscle via direct phosphorylation of PGC-1alpha. Proc Natl Acad Sci USA. 2007;104:12017-22.

16. Yamauchi T, Kamon J, Minokoshi Y, Ito Y, Waki H, Uchida S, et al. Adiponectin stimulates glucose utilization and fatty-acid oxidation by activating AMP-activated protein kinase. Nat Med. 2002;8:1288-95. 
17. Iwabu M, Yamauchi T, Okada-Iwabu M, Sato K, Nakagawa T, Funata M, et al. Adiponectin and AdipoR1 regulate PGC-1alpha and mitochondria by $\mathrm{Ca}(2+)$ and AMPK/SIRT1. Nature. 2010;464:1313-9.

18. Nash MS, Mendez AJ. A guideline-driven assessment of need for cardiovascular disease risk intervention in persons with chronic paraplegia. Arch Phys Med Rehabil. 2007;88:751-7.

19. Bauman WA, Spungen AM. Disorders of carbohydrate and lipid metabolism in veterans with paraplegia or quadriplegia: a model of premature aging. Metabolism. 1994;43:749-56.

20. Buchholz AC, McGillivray CF, Pencharz PB. Differences in resting metabolic rate between paraplegic and able-bodied subjects are explained by differences in body composition. Am J Clin Nutr. 2003;77:371-8.

21. Gorgey AS, Chiodo AE, Zemper ED, Hornyak JE, Rodriguez GM, Gater DR. Relationship of spasticity to soft tissue body composition and the metabolic profile in persons with chronic motor complete spinal cord injury. J Spinal Cord Med. 2010;33:6-15.

22. Castro MJ, Apple DF Jr, Staron RS, Campos GE, Dudley GA. Influence of complete spinal cord injury on skeletal muscle within 6 mo of injury. J Appl Physiol. 1999;86:350-8.

23. Gorgey AS, Dudley GA. Skeletal muscle atrophy and increased intramuscular fat after incomplete spinal cord injury. Spinal Cord. 2007;45:304-9.

24. Wang Y, Huang T, Liang H, Su T, Chen S, Wang T. Fasting serum levels of adiponectin, ghrelin, and leptin in men with spinal cord injury. Arch Phys Med Rehabil. 2005;86:1964-8.

25. Maruyama Y, Mizuguchi M, Yaginuma T, Kusaka M, Yoshida H, Yokoyama K, et al. Serum leptin, abdominal obesity and the metabolic syndrome in individuals with chronic spinal cord injury. Spinal Cord. 2008;46:494-9.

26. O'Brien LC, Wade RC, Segal L, Chen Q, Savas J, Lesnefsky EJ, et al. Mitochondrial mass and activity as a function of body composition in individuals with spinal cord injury. Physiol Rep. 2017a;5:e13080.

27. O'Brien LC, Chen Q, Savas J, Lesnefsky EJ, Gorgey AS. Skeletal muscle mitochondrial mass is linked to lipid and metabolic profile in individuals with spinal cord injury. Eur J Appl Physiol. 2017b;117:2137-47.

28. Gorgey AS, Khalil RE, Gill R, O'Brien LC, Lavis T, Castillo T, et al. Effects of Testosterone and Evoked Resistance Exercise after Spinal Cord Injury (TEREX-SCI): study protocol for a randomised controlled trial. BMJ Open. 2017;7:e014125.

29. Nightingale TE, Gorgey AS. Predicting basal metabolic rate in men with motor complete spinal cord injury. Med Sci Sports Exerc. 2018. https://doi.org/10.1249/MSS.0000000000001548.

30. Gorgey AS, Graham ZA, Bauman WA, Cardozo C, Gater DR. Abundance in proteins expressed after functional electrical stimulation cycling or arm cycling ergometry training in persons with chronic spinal cord injury. J Spinal Cord Med. 2017;40:439-48.

31. Boston RC, Stefanovski D, Moate PJ, Sumner AE, Watanabe RM, Bergman RN. MINMOD Millennium: a computer program to calculate glucose effectiveness and insulin sensitivity from the frequently sampled intravenous glucose tolerance test. Diabetes Technol Ther. 2003;5:1003-15.

32. Gorgey AS, Lawrence J. Acute responses of functional electrical stimulation cycling on the ventilation-to- $\mathrm{CO}_{2}$ production ratio and substrate utilization after spinal cord injury. PM R. 2016;8:225-34.

33. Gorgey AS, Cirnigliaro CM, Bauman W, Adler RA. Estimates of the precision of regional and whole body composition by dual- energy x-ray absorptiometry in persons with chronic spinal cord injury. Spinal Cord. 2018. https://doi.org/10.1038/s41393-0180079-x.

34. Gorgey AS, Mather KJ, Cupp HR, Gater DR. Effects of resistance training on adiposity and metabolism after spinal cord injury. Med Sci Sports Exerc. 2012;44:165-74.

35. Brass EP, Hiatt WR, Gardner AW, Hoppel CL. Decreased NADH dehydrogenase and ubiquinol-cytochrome $\mathrm{c}$ oxidoreductase in peripheral arterial disease. Am J Physiol Heart Circ Physiol. 2001;280:H603-9.

36. Gorgey AS, Gater DR. Demographic of obesity after spinal cord injury. Top Spinal Cord Inj Rehabil. 2007;12:1-7.

37. Gorgey AS, Dolbow DR, Dolbow JD, Khalil RK, Castillo C, Gater DR. Effects of spinal cord injury on body composition and metabolic profile - part I. J Spinal Cord Med. 2014;37:693-702.

38. Rankin KC, O'Brien LC, Segal L, Khan MR, Gorgey AS. Liver adiposity and metabolic profile in individuals with chronic spinal cord injury. Biomed Res Int. 2017;2017:1364818.

39. Mullen K, Pritchard J, Ritchie I, Snook L, Chabowski A, Bonen A, et al. Adiponectin resistance precedes the accumulation of skeletal muscle lipids and insulin resistance in high-fat-fed rats. Am J Physiol Regul Integr Comp Physiol. 2009;296:R243-51.

40. Simmons OL, Kressler J, Nash MS. Reference fitness values in the untrained spinal cord injury population. Arch Phys Med Rehabil. 2014;95:2272-8.

41. Gorgey AS, Poarch HJ, Dolbow DD, Castillo T, Gater DR. Effect of adjusting pulse durations of functional electrical stimulation cycling on energy expenditure and fatigue after spinal cord injury. J Rehabil Res Dev. 2014;51:1455-68.

42. Ruige JB, Ballaux DP, Funahashi T, Mertens IL, Matsuzawa Y, Van Gaal LF. Resting metabolic rate is an important predictor of serum adiponectin concentrations: potential implications for obesity-related disorders. Am J Clin Nutr. 2005;82:21-5.

43. Nevin A, Mayr H, Atresh S, Kemp I, Simmons J, Vivanti A, et al. Feasibility and acceptability of implementing indirect calorimetry into routine clinical care of patients with spinal cord injury. Top Spinal Cord Inj Rehabil. 2016;22:269-76.

44. Jurimae J, Vaiksaar S, Purge P, Jurimae T. Adiponectin and osteocalcin responses to rowing exercise, and the relationship to substrate oxidation in female rowers. Phys Int. 2016;103:220-30.

45. Wu Y, Zhao J, Zhao W, Guo, Bauman S, Cardozo WA, Nandrolone CP. normalizes determinants of muscle mass and fiber type after spinal cord injury. J Neurotrauma. 2012;29:1663-75.

46. Zhou L, Deepa SS, Etzler JC, Ryu J, Mao X, Fang Q, et al. Adiponectin activates AMP-activated protein kinase in muscle cells via APPL1/LKB1-dependent and phospholipase $\mathrm{C} / \mathrm{Ca} 2+/ \mathrm{Ca} 2+/$ calmodulin-dependent protein kinase kinasedependent pathways. J Biol Chem. 2009;284:22426-35.

47. Bruce CR, Mertz VA, Heigenhause GJ, Dyck DJ. The stimulatory effect of globular adiponectin on insulin-stimulated glucose uptake and fatty acid oxidation is impaired in skeletal muscle from obese subjects. Diabetes. 2005;54:3154-60.

48. Martínez-Redondo V, Pettersson AT, Ruas JL. The hitchhiker's guide to PGC-1 $\alpha$ isoform structure and biological functions. Diabetologia. 2015;58:1969-77.

49. Ruas JL, White JP, Rao RR, Kleiner S, Brannan KT, Harrison BC, et al. A PGC- $1 \alpha$ isoform induced by resistance training regulates skeletal muscle hypertrophy. Cell 2012;151:1319-31.

50. Achari AE, Jain SK. Adiponectin, a therapeutic target for obesity, diabetes, and endothelial dysfunction. Int $\mathrm{J}$ Mol Sci. 2017; $18: 1321$. 